\title{
Cosmological Perturbations of a Quartet of Scalar Fields with a Spatially Constant Gradient
}

\author{
Seoktae Koh ${ }^{1}$, Seyen Kouwn ${ }^{2}$, O-Kab Kwon ${ }^{2,3}$, and Phillial $\mathrm{Oh}^{2}$ \\ ${ }^{1}$ Department of Science Education, Jeju National University, Jeju 690-756, South Korea \\ ${ }^{2}$ Department of Physics, BK21 Physics Research Division, Institute of Basic Science, \\ Sungkyunkwan University, Suwon 440-746, Korea \\ ${ }^{3}$ Institute for the Early Universe, Ewha Womans University, Seoul 120-750, South Korea \\ kundol.koh@jejunu.ac.kr, seyen@skku.edu, okabkwon@ewha.ac.kr, ploh@skku.edu
}

\begin{abstract}
We consider the linear perturbations for the single scalar field inflation model interacting with an additional triad of scalar fields. The background solutions of the three additional scalar fields depend on spatial coordinates with a constant gradient $\alpha$ and the ensuing evolution preserves the homogeneity of the cosmological principle. After we discuss the properties of background evolution including an exact solution for the exponential-type potential, we investigate the linear perturbations of the scalar and tensor modes in the background of the slow-roll inflation. In our model with small $\alpha$, the comoving wavenumber has a lower bound $\sim \alpha M_{\mathrm{P}}$ to have well-defined initial quantum states. We find that cosmological quantities, for instance, the power spectrums and spectral indices of the comoving curvature and isocurvature perturbations, and the running of the spectral indices have small corrections depending on the lower bound. Similar behaviors happen for the tensor perturbation with the same lower bound.
\end{abstract}




\section{Contents}

1 Introduction $\quad 2$

2 Background Evolutions $\quad 44$

2.1 Power-law inflation . . . . . . . . . . . . . . . . . . . . 6

2.2 Slow-roll inflation . . . . . . . . . . . . . . . . . . . 8

3 Linear Perturbations $\quad 10$

3.1 Scalar mode . . . . . . . . . . . . . . . . . . . . . . 10

3.1.1 Perturbation equations . . . . . . . . . . . . . . . . 11

3.1.2 Gauge condition . . . . . . . . . . . . . . . . . . 12

3.2 Tensor mode . . . . . . . . . . . . . . . . . . . . . 14

4 Perturbations in the Slow Roll Inflation $\quad 15$

4.1 Scalar mode . . . . . . . . . . . . . . . . . . . . . 16

4.2 Tensor mode . . . . . . . . . . . . . . . . . . . . . . . 20

5 Conclusion 21

\section{Introduction}

Inflation [1,2] has been so far very successful to account for the observational data [3,4]. It is based on the simple idea that the Universe went through a huge accelerating expansion during the early stage of its evolution driven by a single (multiple) scalar field(s). It provides not only spatially flat and homogeneous universe but also a source for small primordial perturbations which are the origin of the large-scale structure of our Universe today. Even though the data are compatible with the single field inflation, there have been considerable interests in multi-field inflation.

From the point of view of cosmological perturbations [5], multi-field inflation exhibits distinctive features of the non-Gaussianity [6,7] and generates isocurvature (entropy) perturbations in addition to adiabatic perturbations of the single scalar field model. There exists an extensive amount of literature how these aspects are incorporated into the specific multi-field inflationary models [8-10]. In particular, the presence of isocurvature modes corresponds to relative perturbations among the various matter fields and affects the final curvature perturbation at the end of inflation by acting as a source term in the evolution equation for the curvature perturbation [10].

So far, most of analysis focused on the time-dependent backgrounds in which each of the scalar field is a function of time only. However, growing interest in this field motivates to seek 
other theoretical possibilities. One of the alternative approaches is to consider spatially dependent backgrounds in cosmology [11,12]. It seems that relatively little attention has been paid to spatially dependent backgrounds in inflation. This might be due to the obstacle which is inherent in these solutions in cosmology: in general, these configurations are not compatible with the cosmological principle of homogeneity and isotropy. However, the conflict can be avoided in the nonlinear sigma models which include a triad of scalar fields, $\sigma^{a}(a=1,2,3)$ It is known that by correlating each scalar field with spatial coordinate, $\sigma^{a} \sim x^{a}$, one can maintain the isotropy and homogeneity in a wide class of field theory models whose Lagrangians are functions of $X=-\frac{1}{2} g^{\mu \nu} \tilde{h}_{a b}(\sigma) \partial_{\mu} \sigma^{a} \partial_{\nu} \sigma^{b}$ without the potential for the $\sigma^{a}$ fields.

The ansatz for scalar fields of a nonlinear sigma model, $\sigma^{m} \sim x^{m}(i=1, \cdots, N)$ with the extra dimension $N$, first appeared in the higher dimensional gravity theory and the solutions of the scalar fields trigger spontaneous compactification of the extra dimensions [13, 14]. The compactified extra space is isomorphic to the target space of scalar fields. A similar ansatz which break the diffeomorphism invariance was used to give masses to gravitons as a Higgs mechanism of gravity [15]. Therefore, the coordinate dependent ansatz in the nonlinear sigma model in gravity theories can be considered as a method of constructing the massive gravity theories [16]. Recently, the four scalar fields were combined into de Sitter target space and used in describing the late-time accelerating universe [17]. See also [18,19] for related topics.

Motivated by the nonlinear sigma models inherited from the higher dimensional gravity theories, in this paper, we consider a nonlinear sigma model with a potential as an action of matter fields in four-dimensional gravity theory,

$$
S=\int d^{4} x \sqrt{-g}\left(\frac{M_{\mathrm{P}}^{2}}{2} R-\frac{1}{2} g^{\mu \nu} \tilde{h}_{m n}(\sigma) \partial_{\mu} \sigma^{m} \partial_{\nu} \sigma^{n}-V(\sigma)\right),
$$

where $m, n=1,2,3,4$ and $M_{\mathrm{P}}$ denotes the Planck mass, $M_{\mathrm{P}} \equiv(8 \pi G)^{-1 / 2}$. Here $\tilde{h}_{m n}$ can be considered as an internal metric of a four-dimensional Riemannian manifold. To construct an inflationary model, we consider a specific choice for the internal metric and potential,

$$
\begin{aligned}
& \tilde{h}_{a b}=f(\varphi) \delta_{a b}, \quad \tilde{h}_{a 4}=0, \quad h_{44}=1, \\
& V(\sigma)=V(\varphi),
\end{aligned}
$$

\footnotetext{
${ }^{1}$ Cosmological perturbations with the triad of scalar fields having solutions $\sigma^{a} \sim x^{a}$ were considered before in [11,12]. The main interest of the paper [11] was to create statistically anisotropic and inhomogeneous perturbations with metric perturbations. On the other hand, in [12] the authors introduced $\mathrm{SO}(3)$ symmetric scalar fields only without potential for the scalar fields. The background solution for the triad of the scalar fields is the same with ours. They considered linear perturbations and non-Gaussianity for the model, dubbed as solid inflation. As we see in (1.1), we additionally considered the inflaton with a potential and find nontrivial results in the linear scalar perturbations.
} 
where $\varphi \equiv \sigma^{4}$ and $f(\varphi)$ is an arbitrary positive function of $\varphi^{2}$. Then the resulting model can be considered as the single scalar field model interacting with a triad of scalar fields $\sigma^{a}$, where the coupling among the inflaton $\varphi$ and the triad of scalar fields is non-minimal, $\sim f(\varphi) X$.

We try to describe the cosmological inflation in terms of the action (1.1). As a background solution with the choice of (1.2), we apply the ansatz $\sigma^{a} \sim x^{a}$ for the background evolution, assuming that other dynamical fields depend on the cosmic time only. As we discussed previously, the resulting cosmological evolution becomes homogeneous and isotropic with the FRW metric. For the background evolution, we find an exact solution describing the power-law inflation and try to figure out the properties of the slow-roll inflation of our model. We consider the linearized scalar and tensor perturbations for the slow-roll inflation with a small spatial constant gradient $\alpha$ for the triad of scalar fields 3 The scalar modes have two degrees of freedom, one from $\varphi$ and the other from the scalar mode of the triad. They are decoupled from each other in the minimal coupling $(f(\varphi)=1)$ and have the form of Sasaki-Mukhanov equation [20] in the spatially flat gauge. We obtain the leading contributions of $\alpha$ for the power spectrums and spectral indices of the comoving curvature and isocurvature perturbations.

One interesting feature of our approach is that in the slow-roll case, the requirement of unitarity for the initial quantum state imposes a lower bound on the comoving wave number. The existence of this limit is a consequence of the nonvanishing aforementioned free parameter $\alpha$ associated with spatial condensations.

The paper is organized as follows. In section 2, we start from the action (1.1) with the choice (1.2). Using the ansatz $\sigma^{a} \sim x^{a}$ for the triad of scalar fields, we obtain an exact solution for the power-law inflation and investigate the properties of the background evolution for the slow-roll inflation. In section 3, we write the perturbed equations for the scalar and tensor modes and discuss the gauge conditions for the scalar perturbation. In section 4, we describe the behaviors of the perturbations up to leading order of $\alpha$ and obtain cosmological quantities. We conclude in section 5 .

\section{Background Evolutions}

We start from the action (1.1) with the choice (1.2),

$$
S=\int d^{4} x \sqrt{-g}\left[\frac{M_{\mathrm{P}}^{2}}{2} R-\frac{1}{2} g^{\mu \nu} \partial_{\mu} \varphi \partial_{\nu} \varphi-\frac{1}{2} f(\varphi) g^{\mu \nu} \partial_{\mu} \sigma^{a} \partial_{\nu} \sigma^{a}-V(\varphi)\right],
$$

\footnotetext{
${ }^{2}$ It is to be pointed out that the four fields have all positive kinetic energy unlike the previously mentioned cases of massive graviton and de Sitter target space where the scalar field $\varphi$ has a negative kinetic energy.

${ }^{3}$ The three perturbed modes of the triad of scalar fields are decomposed into one scalar and two vector modes.
} 
where $\sigma^{a}$ 's have an $\mathrm{SO}(3)$ symmetry 4 This action can be considered as the single field inflation model with a triad of scalar fields having noncanonical kinetic terms. We read the energymomentum tensor as

$$
T_{\mu \nu}=\partial_{\mu} \varphi \partial_{\nu} \varphi+f(\varphi) \partial_{\mu} \sigma^{a} \partial_{\nu} \sigma^{a}-g_{\mu \nu}\left[\frac{1}{2} f(\varphi) g^{\alpha \beta} \partial_{\alpha} \sigma^{a} \partial_{\beta} \sigma^{a}+\frac{1}{2} g^{\alpha \beta} \partial_{\alpha} \varphi \partial_{\beta} \varphi+V(\varphi)\right],
$$

and equations of motion of the scalar fields $\varphi$ and $\sigma^{a}$ as

$$
\begin{aligned}
& \partial_{\mu} \partial^{\mu} \varphi-\frac{1}{2} f^{\prime}(\varphi) g^{\mu \nu} \partial_{\mu} \sigma^{a} \partial_{\nu} \sigma^{a}-V_{\varphi}=0, \\
& \frac{1}{\sqrt{-g}} \partial_{\mu}\left(\sqrt{-g} f(\varphi) g^{\mu \nu} \partial_{\nu} \sigma^{a}\right)=0
\end{aligned}
$$

where $V_{\varphi} \equiv d V / d \varphi$.

The potential in (2.3) only depends on the single scalar field $\varphi$. The reason is related with our choice of the spatially linear background solution for the scalar fields $\sigma^{a}$, which can guarantee the cosmological principle of homogeneity with a $\mathrm{SO}(3)$ invariant potential. As we will see in the next paragraph, the spatially linear solution for $\sigma^{a}$ in the background FRW metric cannot be the solution of the equations of motion (2.5) in the presence of a nonvanishing potential of $\sigma^{a}$. Furthermore, the energy-momentum tensor becomes a function of the spatial coordinates, which means the breakdown of the cosmological principle of homogeneity and isotropy. Therefore, the vanishing of the potential for $\sigma^{a}$ is crucial in this paper.

Now we consider the background evolution in our setup. The background FRW metric is given by

$$
d s^{2}=-d t^{2}+a(t)^{2}\left(d x^{2}+d y^{2}+d z^{2}\right)
$$

where $a(t)$ is the scale factor. In this paper, we consider an ansatz for the scalar field $\sigma^{a}$ as

$$
\sigma^{a}=M_{\mathrm{P}}^{2} \alpha x^{a},
$$

where $\alpha$ is an arbitrary dimensionless constant. Under the assumption that the scalar field $\varphi$ is spatially homogeneous, we easily see that the ansatz (2.7) satisfies the equation of motion for $\sigma^{a}$

\footnotetext{
${ }^{4}$ Our model is also related to the model in 21 in which the authors introduced the inflation scalar field with a potential and a two-form field to explain an anisotropic inflation. This two-form field in four dimensions is dual to a pseudo scalar field.
} 
in (2.5). Remaining equations of motion for $g_{\mu \nu}$ and $\varphi$ are given by

$$
\begin{aligned}
& H^{2}=\frac{\rho}{3 M_{\mathrm{P}}^{2}}, \\
& \dot{H}=-\frac{\rho+p}{2 M_{\mathrm{P}}^{2}}, \\
& \ddot{\varphi}+3 H \dot{\varphi}+\frac{3 M_{\mathrm{p}}^{4} \alpha^{2}}{2 a^{2}} f_{\varphi}+V_{\varphi}=0,
\end{aligned}
$$

where $f_{\varphi} \equiv d f / d \varphi, H \equiv \dot{a} / a$, and

$$
\begin{aligned}
& \rho=\frac{1}{2} \dot{\varphi}^{2}+\frac{3 M_{\mathrm{p}}^{4} \alpha^{2}}{2 a^{2}} f+V, \\
& p=\frac{1}{2} \dot{\varphi}^{2}-\frac{M_{\mathrm{p}}^{4} \alpha^{2}}{2 a^{2}} f-V .
\end{aligned}
$$

As we see from these background equations, the homogeneity of the background evolution is not spoiled though we take the spatially dependent ansatz for $\sigma^{a}$ in (2.7). The $f$-dependent terms in (2.9) are originated from the contributions of $\sigma^{a}$. Therefore, the equation of state of $\sigma$-fields is $w_{\sigma}=-1 / 3$. For concreteness, we fix the function $f(\varphi)$ as

$$
f(\varphi)=e^{2 \xi \varphi / M_{\mathrm{p}}}
$$

where $\xi$ is an arbitrary constant. In the case $\xi=0$, i.e., $f(\varphi)=1$, the role of the $\alpha^{2}$-dependent term in (2.8) is the same as the curvature constant determining the spatial curvature in the Friedmann equations. Since we are considering the positive $\alpha^{2}$, the background evolution under the spatial dependent solution (2.7) corresponds to that of the open universe. However, our model is different from the single field inflation model on the background metric of the open universe. As we will see later, there are nontrivial roles of $\sigma^{a}$ in the perturbation level.

The main purpose of this work is to investigate the behaviors of our model (2.3) from the point of view of linear perturbations. Before we move on to the subject of the perturbation, we describe the background evolutions of our model by considering two inflationary scenarios, the power-law inflation and the slow-roll inflation.

\subsection{Power-law inflation}

It is well-known that under an exponential-type potential the single scalar field model has an exact solution describing the power-law inflation [22]. Comparing with the single scalar field model, there are $\alpha$-dependent terms in (2.8), which are originated from the spatially dependent background solution for $\sigma^{a}$. As we will see in this subsection, the exact solution for the power-law 
inflation in the single scalar field model can be a solution of the equations of motion in (2.8) with some deformations by parameters, $\xi$ and $\alpha$.

As in the case of the single scalar field model, we consider an exponential-type potential

$$
V(\varphi)=V_{0} e^{-\lambda \varphi / M_{\mathrm{P}}}
$$

where $V_{0}$ and $\lambda$ are arbitrary constants. We consider an ansatz which describes the power-law inflation,

$$
a(t)=a_{0}\left(M_{\mathrm{P}} t\right)^{n}, \quad \varphi(t)=M_{\mathrm{P}}\left(\frac{2}{\lambda} \ln \left(M_{\mathrm{P}} t\right)+\varphi_{0}\right),
$$

where $a_{0}, n$, and $\varphi_{0}$ are dimensionless constants.

When $\alpha=0$, the background equations reduce to those of the single scalar field model. The constant parameters in the power-law solution (2.12) are determined as

$$
n=\frac{2}{\lambda^{2}}, \quad \varphi_{0}=\frac{1}{\lambda} \ln \left(\frac{V_{0} \lambda^{4}}{2 M_{\mathrm{P}}^{4}\left(6-\lambda^{2}\right)}\right),
$$

and $a_{0}$ becomes a free parameter.

However, in the case of $\alpha \neq 0$, all constant parameters of the power-law solution (2.12) are fixed by

$$
\begin{aligned}
n & =1+\frac{2 \xi}{\lambda} \\
a_{0}^{2} & =\frac{\alpha^{2} \lambda^{2}}{2\left(\lambda^{2}+2 \lambda \xi-2\right)}\left(\frac{\lambda^{2} V_{0}}{2 M_{\mathrm{P}}^{4}\left(6 \xi^{2}+3 \lambda \xi+2\right)}\right)^{2 \xi / \lambda} \\
\varphi_{0} & =\frac{1}{\lambda} \ln \left(\frac{\lambda^{2} V_{0}}{2 M_{\mathrm{P}}^{4}\left(6 \xi^{2}+3 \lambda \xi+2\right)}\right) .
\end{aligned}
$$

The positive definiteness of the right-hand side of the second condition in (2.14) restricts the value of $\lambda$,

$$
\lambda>-\xi+\sqrt{\xi^{2}+2} \text { or } \quad \lambda<-\xi-\sqrt{\xi^{2}+2} .
$$

In order to have an accelerating unverse, we also have a constraint, $\frac{\xi}{\lambda}>0$, from the first relation in (2.14). Therefore, there are two possibilities for the parameters, $\lambda$ and $\xi$, i.e., $\lambda>0 \& \xi>0$ or $\lambda<0 \& \xi<0$. In the case $\lambda>0 \& \xi>0$ we have the first relation in (2.15), while in the $\lambda<0 \& \xi<0$ case we have the second one in (2.15). Then the range of $n$ for both cases can be expressed in terms of $\xi$ as follows,

$$
1<n<1+\xi^{2}+\sqrt{\xi^{4}+2 \xi^{2}}
$$


In the case $\xi=0, n$ is fixed to unity. Therefore, there is no inflation in this case. In this paper, we consider $\lambda>0 \& \xi>0$ case.

The power-law solutions in (2.13) and (2.14) are special solutions of the background equations of (2.8) since we started from a special ansatz in obtaining the solutions. For this reason, it is understandable that one cannot obtain the solution (2.13) by taking $\alpha \rightarrow 0$ in (2.14). That is, these two solutions satisfy different initial conditions in the $\alpha \rightarrow 0$ limit. The general solution under the exponential-type potential (2.11) for the single scalar field model was obtained in [23].

\subsection{Slow-roll inflation}

Next, we consider the slow-roll inflation with a potential satisfying the slow-roll approximations,

$$
\dot{\varphi}^{2} / 2 \ll V, \quad \ddot{\varphi} \ll 3 H \dot{\varphi} .
$$

To reflect these approximations, we introduce the slow-roll parameters,

$$
\epsilon \equiv \frac{\dot{\varphi}^{2}}{2 M_{\mathrm{P}}^{2} H^{2}}, \quad \eta \equiv \frac{V_{\varphi \varphi}}{3 H^{2}}
$$

With the approximations in (2.17), the background equations of motion of (2.8) reduce to

$$
\begin{aligned}
& H^{2} \simeq \frac{\alpha^{2} M_{\mathrm{P}}^{2}}{2 a^{2}} f+\frac{1}{3 M_{\mathrm{P}}^{2}} V, \\
& 3 H \dot{\varphi}+\frac{3 \alpha^{2} M_{\mathrm{P}}^{4}}{2 a^{2}} f_{\varphi}+V_{\varphi} \simeq 0,
\end{aligned}
$$

where $f_{\varphi}=\frac{2 \xi}{M_{\mathrm{P}}} e^{2 \xi \varphi / M_{\mathrm{P}}}$ for the case (2.10). The background dynamics of $\varphi$ is governed by the two papameters $\alpha$ and $\xi$ as well as the shape of potential $V(\varphi)$.

We can compute the number of $e$-foldings using the slow-roll equations of motion of (2.19),

$$
\mathcal{N}=\int_{t}^{t_{e}} H d t=-\int_{\varphi_{e}}^{\varphi_{i}} \frac{H}{\dot{\varphi}} d \varphi \simeq \frac{1}{M_{\mathrm{P}}^{2}} \int_{\varphi_{e}}^{\varphi_{i}} \frac{\frac{V}{3}+\frac{\alpha^{2} M_{\mathrm{P}}^{4} f}{2 a^{2}}}{\frac{V_{\varphi}}{3}+\frac{\alpha^{2} \xi M_{\mathrm{P}}^{3} f}{a^{2}}} d \varphi
$$

where $\varphi_{i}$ and $\varphi_{e}$ are the values of $\varphi$ at the initial time and at the end of inflation, respectively. For a fixed value of $\alpha$, the number of $e$-foldings decreases with increasing $\xi$ since the $\xi$-dependent term only appears in the denominator in (2.20). For concreteness, we consider a massive scalar field with a potential

$$
V(\varphi)=\frac{1}{2} m^{2} \varphi^{2} .
$$




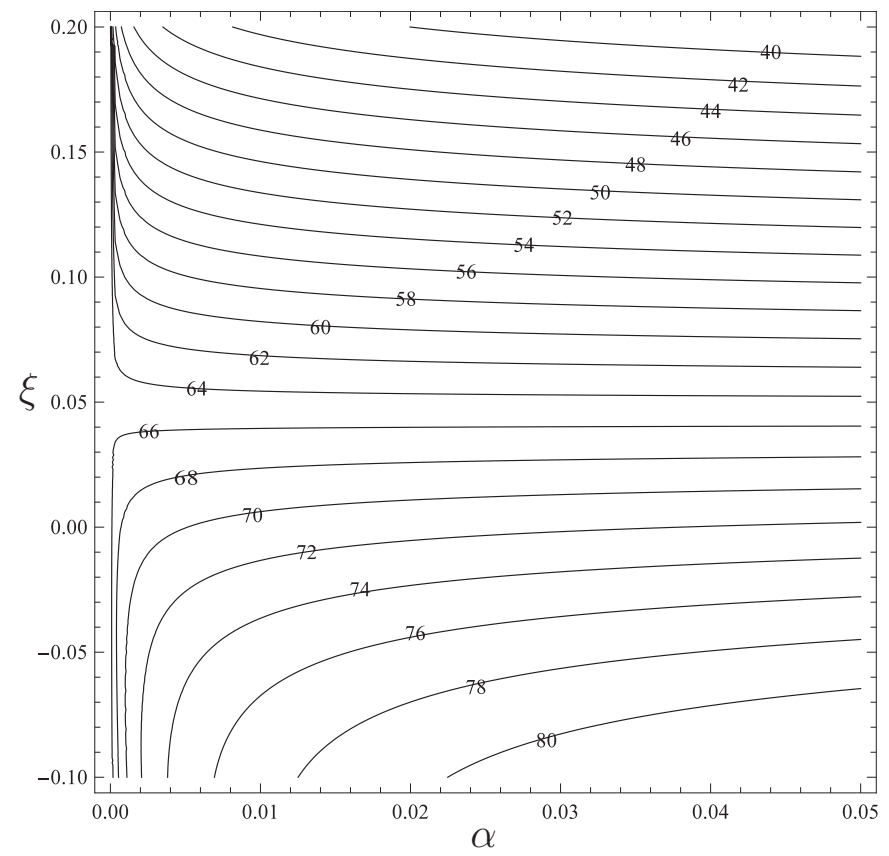

Figure 1: Plots of $e$-foldings for the quadratic potential $V=\frac{1}{2} m^{2} \varphi^{2}$ on the parameter space of $\alpha$ and $\xi$. We choose the initial condition as $a_{0}=1, \varphi_{0}=16 M_{\mathrm{P}}$, and $\dot{\varphi}_{0}=0$ with $m=10^{-6} M_{\mathrm{P}}$.

Then the number of $e$-foldings in (2.20) is given by

$$
\mathcal{N} \simeq \frac{1}{M_{\mathrm{P}}^{2}} \int_{\varphi_{e}}^{\varphi_{i}} \frac{\frac{\varphi}{2}\left(1+\frac{\alpha^{2} M_{\mathrm{P}}^{4} f}{a^{2} m^{2} \varphi^{2}}\right)}{1+\frac{\alpha^{2} \xi M_{\mathrm{P}}^{3} f}{a^{2} m^{2} \varphi}} d \varphi
$$

For a small value of $\alpha$, we can expand the number of $e$-foldings as

$$
\mathcal{N} \simeq \frac{1}{2 M_{\mathrm{P}}^{2}} \int_{\varphi_{e}}^{\varphi_{i}} \varphi\left[1-\left(\xi-\frac{M_{\mathrm{P}}}{\varphi}\right) \frac{\alpha^{2} M_{\mathrm{P}}^{3} f}{a^{2} m^{2} \varphi}+\mathcal{O}\left(\alpha^{4}\right)\right] d \varphi .
$$

In this limit, the $\alpha$-dependent term in (2.23) is also small and the contribution of this term to the number of $e$-foldings is negligible and independent of the magnitude of $\xi$. However, the sign of $\xi-M_{\mathrm{P}} / \varphi$ determines the negative or positive contributions to the number of $e$-foldings. Therefore, there is a critical point for the value of $\xi$ near

$$
\xi \approx M_{\mathrm{P}} / \varphi
$$

The scalar field $\varphi$ is varying as the cosmic time evolves. However, it is almost a constant at the early stage of the inflation, according to our assumption of the slow-roll inflation. For this reason, the value of $\xi$ in (2.24) can be the critical point. The behaviors of the $e$-foldings for the quadratic potential (2.21) are given graphically in Fig.1. 
One of the well-known properties of the slow-roll inflation $(\alpha=0)$ is that there exists a late time attractor behavior, such that the time evolution of the inflaton scalar field becomes independent of the initial conditions. In our case $(\alpha \neq 0)$ in $(2.8)$, the time evolution behavior at the early time is different from that of the single field case due to some contributions of the $\alpha$-dependent terms in (2.8). As time goes on, however, the contributions become smaller quickly since those terms are proportional to $1 / a^{2}$. Therefore, we have similar attractor behavior in our case as well.

\section{Linear Perturbations}

In this section, we discuss the linear perturbations for our model. We introduce a new gauge invariant variable in the scalar perturbation, which is originated from the space dependent background solutions of the triad of scalar fields, $\sigma^{a}$. After we take the spatially flat gauge which is convenient in our later analysis for the linear perturbation, we write the general form of the comoving curvature perturbation and the isocurvature perturbation. We also discuss the perturbation for the tensor mode.

\subsection{Scalar mode}

In this subsection we consider the linear scalar perturbation for our model (2.3). The linear scalar perturbation of the metric is given by

$$
d s^{2}=-(1+2 A) d t^{2}+2 a \partial_{i} B d t d x^{i}+a^{2}\left[(1-2 \psi) \delta_{i j}+2 \partial_{i} \partial_{j} E\right] d x^{i} d x^{j},
$$

where $A, B, \psi$, and $E$ are four scalar perturbation modes. We also consider perturbations of the scalar fields,

$$
\begin{aligned}
\varphi(t, x) & =\varphi(t)+\delta \varphi(t, x), \\
\sigma^{a}(t, x) & =\sigma^{a}(x)+\delta \sigma^{a}(t, x) .
\end{aligned}
$$

Before we write the perturbation equations for scalar modes, we discuss the contribution of $\delta \sigma^{i}$ to scalar modes. At first, we read the $(0, i)$-component of the perturbed Einstein equation $\delta G_{i}^{0}=M_{\mathrm{P}}^{-2} \delta T_{i}^{0}$ as

$$
-2 \partial_{i}(H A+\dot{\psi})=M_{\mathrm{P}}^{-2} \delta T_{i}^{0}=M_{\mathrm{P}}^{-2}\left(-\partial_{i}(\dot{\varphi} \delta \varphi)-\alpha f \delta \dot{\sigma}^{i}+\frac{\alpha^{2} f}{a} \partial_{i} B\right) .
$$

Taking the curl of both sides of (3.27), we obtain

$$
\epsilon_{i j k} \partial^{j} \delta \sigma^{k}=0 .
$$


From this relation, we see that $\delta \sigma^{i}$ appearing in the linear scalar perturbation equations has no contribution to the perpendicular mode. For this reason, we can set5

$$
\delta \sigma^{i}=\frac{1}{k} \partial_{i} u
$$

where $k$ is the comoving wave number and $u$ is the perturbed scalar mode. Here we introduce $1 / k$ factor in order to define the canonical kinetic term for the scalar mode $u$ in the perturbed Lagrangian. Then we can write the variation of the $(0, i)$-component of the energy momentum tensor as

$$
\delta T_{i}^{0}=\partial_{i} \delta q
$$

where the scalar part of the three momentum $\delta q$ is given by

$$
\delta q=-\dot{\varphi} \delta \varphi-\frac{M_{\mathrm{p}}^{2} \alpha}{k} f \dot{u}+\frac{M_{\mathrm{p}}^{4} \alpha^{2}}{a} f B .
$$

\subsubsection{Perturbation equations}

Inserting the relations for perturbed modes, (3.25), (3.26), and (3.29) into the Einstein equation, we obtain the linearized perturbed Einstein equations $\delta G^{\mu}{ }_{\nu}=\frac{1}{M_{\mathrm{P}}^{2}} \delta T_{\nu}^{\mu}$ :

$$
\begin{aligned}
& (0,0): 3 H(H A+\dot{\psi})-\frac{1}{a^{2}} \nabla^{2}\left[\psi+H\left(a^{2} \dot{E}-a B\right)\right]=-\frac{\delta \rho}{2 M_{\mathrm{p}}^{2}}, \\
& (0, i): H A+\dot{\psi}=-\frac{\delta q}{2 M_{\mathrm{p}}^{2}}, \\
& (i, j): 2\left[\ddot{\psi}+3 H(H A+\dot{\psi})+H \dot{A}+2 \dot{H} A+\frac{1}{2 a^{2}} \nabla^{2} D\right] \delta_{j}^{i}-\frac{1}{a^{2}} \partial_{i} \partial_{j} D \\
& =\frac{1}{M_{\mathrm{P}}^{2}}\left(\delta p \delta^{i}{ }_{j}+\Pi^{i}{ }_{j}\right),
\end{aligned}
$$

\footnotetext{
${ }^{5}$ In general, $\delta \sigma^{i}$ is decomposed as $\delta \sigma^{i}=\frac{1}{k} \partial_{i} u+\delta \sigma_{\perp}^{i}$ with the perpendicular mode $\delta \sigma_{\perp}^{i}$ satisfying $\partial_{i} \delta \sigma_{\perp}^{i}=0$ in the linear perturbation. When we consider the perturbation of the vector mode, $\delta \sigma_{\perp}^{i}$ can contribute to the perturbed equations. It is well-known that after the inflation the universe enters into the matter-dominated era, and vector modes should decay leaving no detectable imprints. As we discussed in section 2, the background in our model has similar attractor behavior with that of the single field model. For this reason, the vector modes in our model can decay as well. We do not consider the perturbation of the vector mode in this paper.
} 
where

$$
\begin{aligned}
\delta \rho & =\dot{\varphi}(\dot{\delta} \varphi-\dot{\varphi} A)+\frac{\partial V}{\partial \varphi} \delta \varphi+\frac{M_{\mathrm{p}}^{4} \alpha^{2}}{a^{2}} f\left(\nabla^{2}\left(\frac{u}{\alpha k M_{\mathrm{p}}^{2}}-E\right)+3 \psi+\frac{3 \xi \delta \varphi}{M_{\mathrm{p}}}\right) \\
\delta p & =\dot{\varphi} \delta \dot{\varphi}-A \dot{\varphi}^{2}-V_{\varphi} \delta \varphi-\frac{M_{\mathrm{p}}^{4} \alpha^{2}}{3 a^{2}} f\left(\nabla^{2}\left(\frac{u}{\alpha k M_{\mathrm{p}}^{2}}-E\right)+3 \psi+\frac{3 \xi \delta \varphi}{M_{\mathrm{p}}}\right) \\
D & =A-\psi-H\left(a^{2} \dot{E}-a B\right)-\frac{d}{d t}\left(a^{2} \dot{E}-a B\right) \\
\Pi_{j}^{i} & =\frac{2 M_{\mathrm{P}}^{4} \alpha^{2}}{a^{2}} f\left[\partial_{i} \partial_{j}\left(\frac{u}{\alpha k M_{\mathrm{P}}^{2}}-E\right)-\frac{1}{3} \nabla^{2}\left(\frac{u}{\alpha k M_{\mathrm{P}}^{2}}-E\right)\right] .
\end{aligned}
$$

From (2.4) and (2.5), we obtain the perturbed equations for $\delta \varphi$ and $u$ without gauge fixing,

$$
\begin{aligned}
& \delta \ddot{\varphi}+3 H \delta \dot{\varphi}-\frac{1}{a^{2}} \nabla^{2} \delta \varphi+\frac{2 M_{\mathrm{p}}^{2} \alpha^{2} \xi}{a^{2}} f\left(\frac{\nabla^{2} u}{\alpha k M_{\mathrm{p}}}+3 \xi \delta \varphi\right)+\frac{\partial^{2} V}{\partial \varphi^{2}} \delta \varphi \\
& \quad=2 A \ddot{\varphi}+6 H A \dot{\varphi}+\dot{\varphi}\left[\dot{A}+3 \dot{\psi}+\nabla^{2}\left(\frac{B}{a}-\dot{E}\right)\right]-\frac{2 M_{\mathrm{P}}^{3} \alpha^{2} \xi}{a^{2}} f\left(3 \psi-\nabla^{2} E\right), \\
& \ddot{u}+\left(3 H+\frac{2 \xi \dot{\varphi}}{M_{\mathrm{P}}}\right) \dot{u}-\frac{1}{a^{2}} \nabla^{2} u=\frac{\alpha k M_{\mathrm{P}}^{2}}{a^{2}}\left[a \dot{B}+2 a\left(H+\frac{\xi \dot{\varphi}}{M_{\mathrm{P}}}\right) B+\frac{2 \xi \delta \varphi}{M_{\mathrm{P}}}+\left(A-\psi-\nabla^{2} E\right)\right] .
\end{aligned}
$$

Using the relations in (3.31) and (3.33), we can write the comoving curvature perturbation [24, 25] and the isocurvature perturbation for multiple scalar fields [10] as

$$
\mathcal{R} \equiv \psi-\frac{H}{\rho+p} \delta q, \quad \mathcal{S} \equiv H\left(\frac{\delta p}{\dot{p}}-\frac{\delta \rho}{\dot{\rho}}\right) .
$$

\subsubsection{Gauge condition}

As we see in (3.29), we have one additional scalar degree of freedom due to the contribution of $\delta \sigma^{a}$. Therefore, we have six degrees of freedom for the scalar perturbation modes. Eliminating the gauge degrees of freedom and imposing the constraints of the Einstein equation, we have two physical degrees of freedom. Since we have considered the spatially dependent background solution for $\sigma^{a}$, we have slightly different gauge invariant quantities from the well-known multi-field models.

Under the change of coordinate,

$$
x^{\mu} \rightarrow x^{\mu}+\beta^{\mu}, \quad\left(\beta^{0}=\beta, \beta^{i}=\delta^{i j} \partial_{j} \gamma\right),
$$


the scalar modes of the perturbed metric (3.25) transform as

$$
\begin{aligned}
& A \rightarrow A-\dot{\beta}, \\
& B \rightarrow B+\frac{\beta}{a}-a \dot{\gamma}, \\
& \psi \rightarrow \psi-H \beta \\
& E \rightarrow E-\gamma .
\end{aligned}
$$

The scalar modes of matter fields also transform as

$$
\begin{aligned}
\delta \varphi & \rightarrow \delta \varphi-\beta^{\mu} \partial_{\mu} \varphi=\delta \varphi-\dot{\varphi} \beta \\
\delta \sigma^{a} & \rightarrow \delta \sigma^{a}-\beta^{\mu} \partial_{\mu} \sigma^{a}=\frac{1}{k} \delta^{a b} \partial_{b} u-\alpha M_{\mathrm{P}}^{2} \delta^{a b} \partial_{b} \gamma
\end{aligned}
$$

where we used the background solution $\sigma^{a}=\alpha M_{\mathrm{P}}^{2} x^{a}$ of (2.7) in the last step. From (3.38) we can read off the gauge transformation of the scalar mode $u$ as

$$
u \rightarrow u-k \alpha M_{\mathrm{P}}^{2} \gamma
$$

From these transformation rules, we can obtain several gauge invariant quantities. For later convenience we define those quantities,

$$
\begin{aligned}
\Phi & \equiv A+\frac{d}{d t}(a(B-a \dot{E})), \\
\Psi & \equiv \psi+a H(B-a \dot{E}), \\
Q_{\varphi} & \equiv \delta \varphi-\frac{\dot{\varphi}}{H} \psi, \\
Q_{u} & \equiv u-\alpha k M_{\mathrm{P}}^{2} E .
\end{aligned}
$$

The new quantity $Q_{u}$ was introduced due to the spatial dependence of the background field $\sigma^{a}$.

To solve the perturbed equations (3.32) and (3.26), we take the spatially flat gauge $(\psi=$ $0 \& E=0)$. In this gauge the perturbed equations are reduced to

$$
\begin{gathered}
\ddot{Q}_{\varphi}+3 H \dot{Q}_{\varphi}+\left(\frac{k^{2}}{a^{2}}+\frac{\dot{\varphi} V_{\varphi}}{M_{\mathrm{P}}^{2} H}+V_{\varphi \varphi}+\frac{6 M_{\mathrm{P}}^{2} \alpha^{2} \xi^{2}}{a^{2}} f+\frac{2 M_{\mathrm{P}} \alpha^{2} \xi \dot{\varphi}}{a^{2} H} f\right) Q_{\varphi} \\
-\frac{2 \xi \alpha k M_{\mathrm{P}}}{a^{2}} f Q_{u}+2\left(\frac{\dot{H} \dot{\varphi}}{H}-\ddot{\varphi}\right) A=0, \\
\ddot{Q}_{u}+\left(3 H+\frac{2 \xi \dot{\varphi}}{M_{\mathrm{P}}}\right) \dot{Q}_{u}+\left(\frac{k^{2}}{a^{2}}+\frac{2 \alpha^{2} M_{\mathrm{p}}^{2} f}{a^{2}}\right) Q_{u}-\frac{2 \xi \alpha k M_{\mathrm{P}}}{a^{2}}\left(Q_{\varphi}+a B \dot{\varphi}\right)=0,
\end{gathered}
$$


where $Q_{\varphi}=\delta \varphi, Q_{u}=u$. The scalar modes and $A$ and $B$ satisfy the constraints which are given from the first two equations of (3.32),

$$
\begin{aligned}
& 3 A H^{2}-\frac{k^{2} B H}{a}=\frac{1}{2 M_{\mathrm{p}}^{2}}\left(A \dot{\varphi}^{2}-\dot{\varphi} \dot{Q}_{\varphi}-V_{\varphi} Q_{\varphi}\right)+\left(\alpha k Q_{u}-3 \alpha^{2} \xi M_{\mathrm{p}} Q_{\varphi}\right) \frac{f}{2 a^{2}} \\
& 2 A H=\frac{\dot{\varphi} Q_{\varphi}}{M_{\mathrm{p}}^{2}}+\left(\frac{\alpha}{k} Q_{u}-\frac{\alpha^{2} M_{\mathrm{p}}^{2} B}{a}\right) f .
\end{aligned}
$$

The comoving curvature and isocurvature perturbations of (3.35) are written in the spatially flat gauge as

$$
\begin{aligned}
& \mathcal{R}=H\left[\frac{\dot{\varphi} Q_{\varphi}-\alpha M_{\mathrm{P}}^{2} f\left(\frac{\alpha M_{\mathrm{P}}^{2} B}{a}-\frac{\dot{Q}_{u}}{k}\right)}{\dot{\varphi}^{2}+\frac{\alpha^{2} M_{\mathrm{P}}^{4}}{a^{2}} f}\right], \\
& \mathcal{S}=H\left[\frac{\dot{\varphi}\left(\dot{Q}_{\varphi}-A \dot{\varphi}\right)-V_{\varphi} Q_{\varphi}+\frac{\alpha^{2} M_{\mathrm{P}}^{4}}{3 a^{2}} f\left(\frac{k Q_{u}}{a M_{\mathrm{P}}^{2}}-\frac{3 \xi Q_{\varphi}}{M_{\mathrm{P}}}\right)}{\dot{\varphi}\left(\ddot{\varphi}-V_{\varphi}\right)+\frac{\alpha^{2} M_{\mathrm{P}}^{4}}{a^{2}} f\left(H-\frac{\xi \dot{\varphi}}{M_{\mathrm{P}}}\right)}-\frac{\dot{\varphi}\left(\dot{Q}_{\varphi}-A \dot{\varphi}\right)+V_{\varphi} Q_{\varphi}-\frac{\alpha^{2} M_{\mathrm{P}}^{4}}{a^{2}} f\left(\frac{k Q_{u}}{a M_{\mathrm{P}}^{2}}-\frac{3 \xi Q_{\varphi}}{M_{\mathrm{P}}}\right)}{\dot{\varphi}\left(\ddot{\varphi}+V_{\varphi}\right)-\frac{3 \alpha^{2} M_{\mathrm{P}}^{4}}{a^{2}} f\left(H-\frac{\xi \dot{\varphi}}{M_{\mathrm{P}}}\right)}\right] .
\end{aligned}
$$

In the case $\alpha=0$, the quantities $\mathcal{R}$ and $\mathcal{S}$ are reduced to those of the single scalar field [10].

\subsection{Tensor mode}

In this subsection, we investigate the linear perturbation of the tensor mode for our model on the background described by the equations of (2.7) and (2.8). Since the tensor mode is decoupled from the scalar and vector ones in the linear perturbation theory, we consider the perturbed metric in the conformal time to describe the tensor perturbation,

$$
d s^{2}=a^{2}(\tau)\left(-d \tau^{2}+\left(\delta_{i j}+h_{i j}\right) d x^{i} d x^{j}\right)
$$

where $d t=a d \tau$. The tensor modes $h_{i j}$ satisfy the following conditions,

$$
\partial_{i} h_{i j}=0, \quad h_{i i}=0
$$

Since the tensor modes $h_{i j}$ have two degrees of freedom, which are identified with the two polarizations of gravitational waves, we expand the tensor modes as

$$
h_{i j}(\tau, \vec{x})=\frac{2}{M_{\mathrm{P}}} \sum_{\lambda=+,-} \frac{\tilde{\mu}_{\lambda}(\tau, \vec{x})}{a} \epsilon_{i j}^{\lambda},
$$


where $\epsilon_{i j}^{\lambda}$ is the polarization tensor satisfying the orthogonality condition $\epsilon_{i j}^{\lambda} \epsilon_{\lambda^{\prime}}^{i j}=\delta_{\lambda^{\prime}}^{\lambda}$. Inserting (3.44) and (3.46) into the action (2.3) on our background and solving the equation of the motion of $\tilde{\mu}_{\lambda}$, we see that the Fourier mode of $\tilde{\mu}_{\lambda}$ satisfies

$$
\mu_{\lambda}^{\prime \prime}(\tau ; k)+\left(k^{2}-\frac{a^{\prime \prime}}{a}\right) \mu_{\lambda}(\tau ; k)=0,
$$

where $^{\prime} \equiv d / d \tau$ and $\mu_{\lambda}$ satisfies the normalization condition,

$$
\mu_{\lambda} \mu_{\lambda}^{\prime *}-\mu_{\lambda}^{*} \mu_{\lambda}^{\prime}=i
$$

Using the relations for the background evolution in (2.8), we obtain a general differential equation for the tensor mode for our case,

$$
\mu_{\lambda}^{\prime \prime}+\left(k^{2}-\frac{1}{2} M_{\mathrm{P}}^{2} \alpha^{2} f+\frac{1}{6 M_{\mathrm{P}}^{2}}\left(\varphi^{\prime 2}-4 a^{2} V\right)\right) \mu_{\lambda}=0 .
$$

\section{Perturbations in the Slow Roll Inflation}

As we see from the perturbed equations (3.41) and (3.42) in the spatially flat gauge, the perturbed modes $Q_{\varphi}$ and $Q_{u}$ are completely decoupled in the $\alpha \rightarrow 0$ limit. Moreover, the resulting curvature perturbations, $\mathcal{R}$ and $\mathcal{S}$ in (3.43), are independent of $Q_{u}$. Therefore, in the $\alpha \rightarrow 0$ limit, the linear perturbation of our model is the same as that of the single scalar field model. For this reason, in this paper, we only consider the case with nonvanishing $\alpha$.

When we consider the linear perturbations with nonvanishing $\xi$ and $\alpha$, in general the modes $Q_{\varphi}$ and $Q_{u}$ are not decoupled. However, in this coupled case it is very difficult to obtain some cosmological quantities, such as power spectrums and scalar/tensor spectral indices. We leave this general case for further investigation. In this paper, we restrict the linear perturbations to the case of the slow-roll inflation at a small value of $\alpha$ with vanishing $\xi$. As we will see in this case, the modes $Q_{\varphi}$ and $Q_{u}$ are decoupled. However, $\mathcal{R}$ and $\mathcal{S}$ still have dependence on both $Q_{\varphi}$ and Qu.

In this paper, we will not consider the perturbation for the power-law inflation. The reason is the following. For the case of $\alpha \neq 0$ with $\xi=0$, the background equations in (2.8) have a special solution given in (2.12) and (2.14) for the exponential-type potential (2.11). In this case, the solutions are reduced to

$$
\varphi=\frac{2 M_{\mathrm{P}}}{\lambda} \ln \left(\frac{\sqrt{V_{0}} \lambda t}{2 M_{\mathrm{P}}}\right), \quad a(t)=\frac{M_{\mathrm{P}} \alpha \lambda}{\sqrt{2 \lambda^{2}-4}} t .
$$

The scale factor is a linear function of the cosmic time $t$, and so accelerating universe is not allowed. Presumably, if we can obtain the general solution like the work for the single scalar with 
exponential-type potential in [23], we may have an inflation era even in the case $\xi=0$. However, it is beyond the range of this paper. Due to this reason, we do not consider the cosmological perturbation for the power-law inflation. In this section, we concentrate on the perturbation for the slow-roll inflation described in section 2.

\subsection{Scalar mode}

In the slow-roll approximation at a small value of $\alpha$ with $\xi=0$, the perturbed equation (3.41) are reduced to

$$
\begin{aligned}
& \ddot{Q}_{\varphi}+3 H \dot{Q}_{\varphi}+\left(\frac{k^{2}}{a^{2}}+\frac{\dot{\varphi} V_{\varphi}}{M_{\mathrm{P}}^{2} H}+V_{\varphi \varphi}\right) Q_{\varphi} \simeq 0, \\
& \ddot{Q}_{u}+3 H \dot{Q}_{u}+\left(\frac{k^{2}}{a^{2}}+\frac{2 \alpha^{2} M_{\mathrm{p}}^{2}}{a^{2}}\right) Q_{u} \simeq 0,
\end{aligned}
$$

where we keep the leading order in slow-roll parameters of (2.18). Changing the cosmic time $t$ into the conformal time $\tau$ and introducing the Sasaki-Mukhanov variables,

$$
\mathcal{V}=a Q_{\varphi}, \quad \mathcal{U}=a Q_{u}
$$

we obtain the Sasaki-Mukhanov equations from (4.51),

$$
\begin{aligned}
& \mathcal{V}_{k}^{\prime \prime}+\left(k_{1}^{2}-\frac{\mu_{1}^{2}-\frac{1}{4}}{\tau^{2}}\right) \mathcal{V}_{k}=0 \\
& \mathcal{U}_{k}^{\prime \prime}+\left(k_{2}^{2}-\frac{\mu_{2}^{2}-\frac{1}{4}}{\tau^{2}}\right) \mathcal{U}_{k}=0
\end{aligned}
$$

The parameters in (4.53) are given by

$$
\begin{aligned}
& k_{1}^{2} \equiv k^{2}-\frac{\alpha^{2} M_{\mathrm{P}}^{2}}{6} \\
& k_{2}^{2} \equiv k^{2}+\frac{11 \alpha^{2} M_{\mathrm{P}}^{2}}{6}, \\
& \mu_{1} \simeq \frac{3}{2}+3 \epsilon-\eta \\
& \mu_{2} \simeq \frac{3}{2}+\epsilon
\end{aligned}
$$

The $k_{1}$ and $k_{2}$ can be considered as the effective wavevectors for the $\mathcal{V}_{k}$ and $\mathcal{U}_{k}$ modes, respectively. In driving the Sasaki-Mukhanov equations in (4.53), we used the background equations (2.19) in 
the slow-roll approximation of the subsection 2.2. The conformal time $\tau$ has the following relation

$$
\tau=\int \frac{d t}{a}=-\frac{1}{a H}+\int \frac{\epsilon^{H}}{a^{2} H} d a \simeq-\frac{1}{a H}\left(1+\epsilon+\frac{\alpha^{2} M_{\mathrm{P}}^{2}}{6 a^{2} H^{2}}\right),
$$

where the relation

$$
\epsilon^{H}=-\frac{\dot{H}}{H^{2}} \simeq \epsilon+\frac{\alpha^{2} M_{\mathrm{P}}^{2}}{2 a^{2} H^{2}},
$$

was used. Here we keep the leading order in the slow-roll parameters, $\epsilon$ and $\eta$, and a small gradient constant $\alpha$. We will discuss the range of $\alpha$ in our approximation later. Then, from (4.55), we obtain

$$
\mathcal{H}^{2} \simeq \frac{1+2 \epsilon}{\tau^{2}}+\frac{\alpha^{2} M_{\mathrm{P}}^{2}}{3}
$$

where $\mathcal{H}=a^{\prime} / a$.

Assuming that the slow-roll parameters are constants, we have the exact solutions for the equations of (4.53),

$$
\begin{aligned}
& \mathcal{V}_{k}=\sqrt{-\tau}\left[c_{1 k} H_{\mu_{1}}^{(1)}\left(-k_{1} \tau\right)+d_{1 k} H_{\mu_{1}}^{(2)}\left(-k_{1} \tau\right)\right] e_{1}(k), \\
& \mathcal{U}_{k}=\sqrt{-\tau}\left[c_{2 k} H_{\mu_{1}}^{(1)}\left(-k_{2} \tau\right)+d_{2 k} H_{\mu_{2}}^{(2)}\left(-k_{2} \tau\right)\right] e_{2}(k),
\end{aligned}
$$

where $H_{\mu}^{(i)}(z)(i=1,2)$ are the first and second kinds of the Hankel functions, and $e_{i}(k)$ 's are independent Gaussian random variables satisfying

$$
\left\langle e_{i}(k)\right\rangle=0, \quad\left\langle e_{i}(k) e_{j}\left(k^{\prime}\right)\right\rangle=\delta_{i j} \delta\left(k-k^{\prime}\right)
$$

Here, the angled brackets denote ensemble averages.

In order to determine the integration constants $c_{i k}$ and $d_{i k}$, we have to fix the initial vacuum state at $k \tau \rightarrow-\infty$. As we see in (4.53) and (4.58), the two differential equations and the corresponding solutions have the same forms. Due to the overall normalization of $u$ mode given in (3.29), $\mathcal{V}_{k}$ and $\mathcal{U}_{k}$ modes have the same normalizations. Therefore, we can concentrate on the solution of $\mathcal{V}_{k}$ only and apply the result to the case of $\mathcal{U}_{k}$.

The mode for $\mathcal{V}_{k} \equiv \tilde{v}_{k} e_{1}(k)$ satisfies the normalization condition, $\tilde{v}_{k} \tilde{v}_{k}^{*^{\prime}}-\tilde{v}_{k}^{*} \tilde{v}_{k}^{\prime}=i$ which is originated from the quantization condition of the mode. Due to this normalization, we have the relation between the integration constants,

$$
\left|c_{1 k}\right|^{2}-\left|d_{1 k}\right|^{2}=\frac{\pi}{4}
$$


We adopt the Bunch-Davies vacuum for the initial perturbation mode at $\tau \ll 0$ by taking the positive energy mode. Then the initial mode is given by

$$
\mathcal{V}_{k}(\tau)=\frac{1}{\sqrt{2 k_{1}}} e^{-i k_{1} \tau} e_{1}(k)
$$

This mode corresponds to the choice of coefficients,

$$
c_{1 k}=e^{\frac{i \pi}{2}\left(\nu+\frac{1}{2}\right)} \frac{\sqrt{\pi}}{2}, \quad d_{1 k}=0 .
$$

Using the relations (4.61) and (4.62), and adopting to the case of $\mathcal{U}_{k}$ as well, we have the exact solutions for $\mathcal{V}_{k}$ and $\mathcal{U}_{k}$,

$$
\begin{aligned}
& \mathcal{V}_{k}(\tau)=\frac{\sqrt{\pi}}{2} e^{\frac{i \pi}{2}\left(\mu_{1}+\frac{1}{2}\right)} \sqrt{-\tau} H_{\mu_{1}}^{(1)}\left(-k_{1} \tau\right) e_{1}(k), \\
& \mathcal{U}_{k}(\tau)=\frac{\sqrt{\pi}}{2} e^{\frac{i \pi}{2}\left(\mu_{2}+\frac{1}{2}\right)} \sqrt{-\tau} H_{\mu_{2}}^{(1)}\left(-k_{2} \tau\right) e_{2}(k) .
\end{aligned}
$$

As we see in the initial mode (4.61), the effective wave number $k_{1}$ should be real to have a well-defined quantum state. This means that the square of the effective wave number $k_{1}^{2}$ should be non-negative. Therefore, the wave number $k$ should be constrained from (4.54) as

$$
k^{2} \geq k_{\min }^{2} \equiv \frac{\alpha^{2} M_{\mathrm{P}}^{2}}{6}
$$

As we see in (4.53), the initial quantum fluctuation modes of $\mathcal{V}_{k}$ with $|k|<\left|k_{\min }\right|$ are exponentially growing or decreasing and so they break the unitary symmetry of the quantum system in the deep inside of the horizon. That is, these modes are not allowed as initial quantum modes. Therefore, there is an observational lower bound of the comoving wavenumber in our model. In the large scale limit $k_{1,2}|\tau| \ll 1$, the modes in (4.63) can be approximated as

$$
\begin{aligned}
& \mathcal{V}_{k}(\tau) \simeq e^{\frac{i \pi}{2}\left(\mu_{1}+\frac{1}{2}\right)} 2^{\mu_{1}-\frac{3}{2}} \frac{\Gamma\left(\mu_{1}\right)}{\Gamma\left(\frac{3}{2}\right)} \frac{1}{\sqrt{2 k_{1}}}\left(-k_{1} \tau\right)^{\frac{1}{2}-\mu_{1}} e_{1}(k), \\
& \mathcal{U}_{k}(\tau) \simeq e^{\frac{i \pi}{2}\left(\mu_{2}+\frac{1}{2}\right)} 2^{\mu_{2}-\frac{3}{2}} \frac{\Gamma\left(\mu_{2}\right)}{\Gamma\left(\frac{3}{2}\right)} \frac{1}{\sqrt{2 k_{2}}}\left(-k_{2} \tau\right)^{\frac{1}{2}-\mu_{2}} e_{2}(k)
\end{aligned}
$$

In the slow-roll approximation with $\xi=0$ and a small nonvanishing value of $\alpha$, the leading contributions for the curvature and isocurvature perturbations in (3.43) are given by

$$
\begin{aligned}
& \mathcal{R} \simeq \frac{Q_{\varphi}}{\sqrt{2 \epsilon} M_{\mathrm{P}}}+\frac{\alpha}{2 k \epsilon H} \dot{Q}_{u}-\frac{\alpha^{2} M_{\mathrm{P}}}{2 \sqrt{2} \epsilon^{3 / 2} a^{2} H^{2}} Q_{\varphi} \\
& \mathcal{S} \simeq \frac{\sqrt{2}}{3 \sqrt{\epsilon} M_{\mathrm{P}} H} \dot{Q}_{\varphi}-\frac{\alpha k}{9 \epsilon a^{2} H^{2}} Q_{u}+\frac{\alpha^{2} M_{\mathrm{P}}}{a^{2} H^{2}}\left(\frac{Q_{\varphi}}{3 \sqrt{2} \epsilon^{3 / 2}}-\frac{\sqrt{2}}{9 \epsilon^{3 / 2} H} \dot{Q}_{\varphi}\right) .
\end{aligned}
$$


From these expressions, we obtain power spectrums for $\mathcal{R}$ and $\mathcal{S}$,

$$
\begin{aligned}
& \mathcal{P}_{\mathcal{R}}(k) \equiv \frac{k^{3}}{2 \pi^{2}}\left\langle\mathcal{R} \mathcal{R}^{*}\right\rangle \simeq \frac{k^{3}}{4 \pi^{2} \epsilon M_{\mathrm{P}}^{2}}\left(1-\frac{\alpha^{2} M_{\mathrm{P}}^{2}}{a^{2} H^{2} \epsilon}\right)\left\langle Q_{\varphi} Q_{\varphi}^{*}\right\rangle, \\
& \mathcal{P}_{\mathcal{S}}(k) \equiv \frac{k^{3}}{2 \pi^{2}}\left\langle\mathcal{S} \mathcal{S}^{*}\right\rangle \simeq \frac{k^{3} \alpha^{2}}{2 \pi^{2} \epsilon^{2}}\left[\frac{k^{2}}{81 a^{4} H^{4}}\left\langle Q_{u} Q_{u}^{*}\right\rangle+\frac{1}{9 a^{2} H^{3}}\left(\left\langle Q_{\varphi} \dot{Q}_{\varphi}^{*}\right\rangle+\left\langle\dot{Q}_{\varphi} Q_{\varphi}^{*}\right\rangle\right)\right] .
\end{aligned}
$$

Using the relations of (4.52) and (4.65), we obtain the power spectrums at the horizon crossing point $(k=a H)$ as

$$
\begin{aligned}
& \mathcal{P}_{\mathcal{R}_{*}} \simeq \frac{H_{*}^{2}}{8 \pi^{2} M_{\mathrm{P}}^{2}} \frac{1}{\epsilon}\left(1+(2-2 C) \eta+(6 C-8) \epsilon-\frac{M_{\mathrm{P}}^{2}}{k^{2}} \frac{\alpha^{2}}{\epsilon}\right), \\
& \mathcal{P}_{\mathcal{S}_{*}} \simeq\left(\frac{H_{*}}{18 \pi}\right)^{2} \frac{\alpha^{2}}{\epsilon^{2} k^{2}}\left(1+2 C-32 \epsilon-18 \eta-\frac{73 \alpha^{2} M_{\mathrm{P}}^{2}}{12 k^{2}}\right),
\end{aligned}
$$

where the subscripted asterisk indicates the value at the horizon crossing point and $C=2-\ln 2-\gamma$ with the Euler-Mascheroni constant $\gamma \approx 0.5772$. In the computations of power spectrums in (4.69), we have used the relations,

$$
\begin{aligned}
& \left\langle Q_{\varphi} Q_{\varphi}^{*}\right\rangle_{*}=\frac{1}{a_{*}^{2}}\left\langle\mathcal{V}_{k} \mathcal{V}^{*}\right\rangle_{*} \simeq \frac{H_{*}^{2}}{2 k^{3}}\left(1+(6 C-8) \epsilon+(2-2 C) \eta-\frac{\alpha^{2} M_{\mathrm{P}}^{2}}{12 k^{2}}\right), \\
& \left\langle Q_{\varphi} \dot{Q}_{\varphi}^{*}\right\rangle+\left\langle\dot{Q}_{\varphi} Q_{\varphi}^{*}\right\rangle=\frac{1}{a_{*}^{3}}\left(\left\langle\mathcal{V}_{k} \mathcal{V}_{k}^{*}\right\rangle_{*}+\left\langle\mathcal{V}_{k}^{\prime} \mathcal{V}_{k}^{*}\right\rangle_{*}\right)-\frac{2 H_{*}}{a_{*}^{2}}\left\langle\mathcal{V}_{k} \mathcal{V}_{k}^{*}\right\rangle_{*} \simeq \frac{H_{*}^{3}}{k^{3}}\left(2 \epsilon-\eta-\frac{\alpha^{2} M_{\mathrm{P}}^{2}}{6 k^{2}}\right) \\
& \left\langle Q_{u} Q_{u}^{*}\right\rangle=\frac{1}{a_{*}^{2}}\left\langle\mathcal{U}_{k} \mathcal{U}_{k}^{*}\right\rangle_{*} \simeq \frac{H_{*}^{2}}{2 k^{3}}\left(1+2(C-2) \epsilon-\frac{37 \alpha^{2} M_{\mathrm{P}}^{2}}{12 k^{2}}\right)
\end{aligned}
$$

The expansions of power spectrums in (4.69) are only valid in the range of $\alpha$,

$$
M_{\mathrm{P}}^{2} \alpha^{2} \lesssim \epsilon^{2} k^{2}
$$

In $\alpha \rightarrow 0$ limit, the power spectrums $\mathcal{P}_{\mathcal{R}}$ and $\mathcal{P}_{\mathcal{S}}$ at the horizon crossing point in (4.69) become those of the single field case. Finally, the spectral indices for $\mathcal{R}$ and $\mathcal{S}$ in the large scale limit $\left(k_{1,2}|\tau| \ll 1\right)$ are given by

$$
\begin{aligned}
& n_{\mathcal{R}}-1 \equiv \frac{d \ln \mathcal{P}_{\mathcal{R}}}{d \ln k} \simeq 2 \eta-6 \epsilon+\frac{2 \alpha^{2} M_{\mathrm{P}}^{2}}{\epsilon k^{2}} \\
& n_{\mathcal{S}}-1 \equiv \frac{d \ln \mathcal{P}_{\mathcal{R}}}{d \ln k} \simeq-2+4 \eta-10 \epsilon+\frac{67 \alpha^{2} M_{\mathrm{P}}^{2}}{6 k^{2}}
\end{aligned}
$$

If we take (4.71) into account, we find the last terms in $n_{\mathcal{R}}-1$ and $n_{\mathcal{S}}-1$ are of the order of $\epsilon$ and $\epsilon^{2}$, respectively. These imply that while the curvature perturbation is nearly scale invariant, the isocurvature perturbation is proportional to the inverse square of the comoving wavenumber. 
The running of the scalar spectral index is given by

$$
\frac{d n_{\mathcal{R}}}{d \ln k}=-\frac{4 \alpha^{2} M_{\mathrm{P}}^{2}}{\epsilon k^{2}}+\mathcal{O}\left(\epsilon^{2}, \epsilon \eta\right)
$$

The leading term becomes of the order of $\epsilon$ from (4.69) and (4.71). Recent Planck [3] and WMAP nine-year [4] data show

$$
\begin{aligned}
& n_{\mathcal{R}}=0.9603, \quad \frac{d n_{\mathcal{R}}}{d \ln k}=-0.0134 \quad(\text { Planck }), \\
& n_{\mathcal{R}}=0.9608, \quad \frac{d n_{\mathcal{R}}}{d \ln k}=-0.019 \quad(\text { WMAP }-9 \mathrm{yr})
\end{aligned}
$$

with the pivot scale $k_{0}=0.05 \mathrm{Mpc}^{-1}$ (Planck) and $k_{0}=0.002 \mathrm{Mpc}^{-1}$ (WMAP), respectively. Since $\alpha^{2}>0$ and $\epsilon>0$, the sign of the running of the spectral index in (4.73) is consistent with the observations. However, to give some restriction on the value of $\alpha$ we need more investigations using a specific potential of the single scalar field. We leave these issues for future work.

\subsection{Tensor mode}

Using the relations (4.56) and (4.57), we obtain

$$
\frac{a^{\prime \prime}}{a}=(a H)^{2}\left(2+\frac{\dot{H}}{H^{2}}\right) \simeq \mathcal{H}^{2}\left(2-\epsilon^{H}\right) \simeq \frac{2+3 \epsilon}{\tau^{2}}+\frac{\alpha^{2} M_{\mathrm{P}}^{2}}{6}
$$

Then the linearized perturbed equation (3.47) for the tensor mode, up to the leading order in the slow-roll parameters and the gradient constant $\alpha$, can be written as

$$
u_{T}^{\prime \prime}+\left[k_{T}^{2}-\frac{\mu_{T}^{2}-\frac{1}{4}}{\tau^{2}}\right] u_{T}=0,
$$

where

$$
k_{T}^{2}=k^{2}-\frac{\alpha^{2} M_{\mathrm{P}}^{2}}{6}, \quad \mu_{T}=\frac{3}{2}+\epsilon .
$$

Interestingly, the tensor mode has the same lower bound of the comoving wavenumber with that of the scalar mode in (4.64).

Choosing the initial condition with a positive frequency mode, just like (4.61) in the initial state of the scalar mode, we obtain the following solutions in the large scale limit $\left(k_{T}|\tau| \ll 1\right)$,

$$
\begin{aligned}
u_{T} & \simeq \frac{\sqrt{\pi}}{2} e^{\frac{i \pi}{2}\left(\mu_{T}+\frac{1}{2}\right)} \sqrt{-\tau} H_{\mu_{T}}^{(1)}\left(-k_{T} \tau\right) e_{T}(k), \\
& \simeq e^{i\left(\mu_{T}+\frac{1}{2}\right) \frac{\pi}{2}} \frac{1}{\sqrt{2 k_{T}}} A(\epsilon)\left(-k_{T} \tau\right)^{-1} e_{T}(k),
\end{aligned}
$$


where $A(\epsilon)=1-\epsilon \ln (-k \tau)+\epsilon(2-\gamma-\ln 2)$ and $e_{T}(k)$ is an independent Gaussian random variable and has the same properties with those defined in (4.59).

With these solutions, the power spectrum for the tensor modes $h_{i j}$ is obtained as

$$
\mathcal{P}_{T}(k)=2 \mathcal{P}_{h}=\frac{8}{a^{2} M_{\mathrm{P}}^{2}} \mathcal{P}_{u_{T}}=\frac{2 H_{*}^{2}}{M_{\mathrm{P}}^{2} \pi^{2}}\left(1+(2 C-4) \epsilon-\frac{\alpha^{2} M_{\mathrm{P}}^{2}}{12 k^{2}}\right),
$$

where we used $h_{\lambda}=\frac{2}{a M_{\mathrm{P}}} u_{T}$ and the factor " 2 " comes from the two polarization states. We also used the quantities at the horizon crossing point $(k=a H)$,

$$
\begin{aligned}
& \left.\left(-k_{T} \tau\right)^{n}\right|_{*} \simeq\left(1+\epsilon+\frac{\alpha^{2} M_{\mathrm{P}}^{2}}{12 k^{2}}\right)^{n}, \\
& \left\langle u_{T} u_{T}^{*}\right\rangle_{*} \simeq \frac{1}{2 k}\left(1+(2 C-4) \epsilon-\frac{\alpha^{2} M_{\mathrm{P}}^{2}}{12 k^{2}}\right) .
\end{aligned}
$$

The spectral index for tensor modes and tensor-to-scalar ratio are given by

$$
\begin{aligned}
& n_{T}-1 \equiv \frac{d \ln \mathcal{P}_{\mathcal{T}}}{d \ln k} \simeq-2 \epsilon-\frac{5 \alpha^{2} M_{\mathrm{P}}^{2}}{6 k^{2}} \\
& r \equiv \frac{\mathcal{P}_{T}}{\mathcal{P}_{\mathcal{R}}} \simeq 16 \epsilon_{*}\left(1+(4-4 C) \epsilon+(2 C-2) \eta+\frac{\alpha^{2} M_{\mathrm{P}}^{2}}{k^{2} \epsilon}\right) .
\end{aligned}
$$

\section{Conclusion}

In this paper, we studied the cosmological properties of a multi-field inflation model in which we considered the single field and an additional triad of scalar fields with a non-minimal coupling which is dependent on the single scalar field.

As a special background solution, we considered spatially dependent linear profiles for the triad of scalar fields, while the single scalar field depends on the cosmic time only. Due to this linear property, all components of the energy momentum tensor evolve homogeneously and isotropically on the background FRW metric. For this reason, we can regard the background evolution as that of the single field inflation in the presence of a matter with an equation of state $w=-1 / 3$, which

spreads to the whole space. However, there appeared one more scalar degree of freedom from the triad of scalar fields in the perturbation level. Therefore, our model is different form the single field inflation model in some cosmological background.

For the exponential-type potential, we found an exact solution describing the power-law inflation, as in the case of the single field inflation model. We also investigated the properties of the background evolution under the assumption of the slow-roll inflation. The background behaviors of the slow-roll inflation are determined by the two parameters $\xi$ and $\alpha$ which are associated with the non-minimal coupling and the gradient constant of the triad of scalar fields, respectively. We 
have drawn contour lines of the $e$-foldings for the quadratic potential on the parameter space of $\xi$ and $\alpha$ in Fig.1.

We also investigated the properties of the scalar and tensor perturbations for the slow-roll inflation with vanishing $\xi$ and small $\alpha$. In the scalar fluctuations, there are two physical modes, $\mathcal{V}_{k}$ and $\mathcal{U}_{k}$ originating from the scalar field and the triad of scalar fields respectively in the spatially flat gauge. The two scalar modes are decoupled from each other and have the form of SasakiMukhanov equation in the leading contribution of $\alpha$. We found that there exists an observational lower bound of the comoving wave number, $\left|k_{\min }\right| \sim \alpha M_{\mathrm{P}}$, to have a well-defined initial quantum state for $\mathcal{V}_{k}$ with a positive energy eigenvalue. We also obtained the power spectrums and the corresponding spectral indices for the comoving curvature and isocurvature perturbations. If we compare our results for the power spectrum with those of the single field inflation model, the $\alpha$-dependent terms are always proportional to $\alpha^{2} / k^{2}$. Due to this reason, the spectral indices for the curvature and isocurvature perturbations have the contribution of $\alpha^{2} / k^{2}$ terms as well. As is already well-known, the isocurvature perturbation in the single field inflation model is vanishing in the large scale. However, the power spectrum for the isocurvature perturbation is non-vanishing and has contributions from both $\mathcal{V}_{k}$ and $\mathcal{U}_{k}$ modes in our model. We also implemented the same analysis for the tensor perturbation. We found the same lower bound of the comoving wavenumber in the tensor mode.

In the case of non-vanishing $\xi, \mathcal{V}_{k}$ and $\mathcal{U}_{k}$ modes are coupled with each other. Then it is difficult to obtain analytic solutions, unlike the case of $\xi=0$. As we saw in the analysis of the background evolution, the value of $\xi$ changes the behaviors of the cosmological evolution significantly. Therefore, we can expect that the value $\xi$ may have some important roles in the perturbations as well. We leave the analysis of the nonvanishing $\xi$ for future work.

One important difference between the single field model and ours is the existence of the isocurvature perturbation in our model, which originates from the spatial gradient of the background solution. One may also test whether the nonvanishing spatial gradient can be a source of the cosmological non-Gaussianity. In the solid inflation model [12, the authors obtained unusually large non-Gaussianity, $f_{N L} \sim 1 /\left(\epsilon c_{s}^{2}\right)$ with sound speed $c_{s}$. Since we are considering the inflaton field in addition to the triad of the scalar fields, we can expect that the non-Gaussianity behaviors of our model is different from that of the solid inflation model. It will be interesting if we compare the non-Gaussianity behavior of our model with those of the solid inflation model and the recent observational data. The details will be reported elsewhere. 


\section{Acknowledgements}

We would like to thank Inyong Cho, Shinsuke Kawai, Eric V. Linder, Seong Chan Park, and Uros Seljak for helpful discussions. This research was supported by Basic Science Research Program through the National Research Foundation of Korea (NRF) funded by the Ministry of Education, Science and Technology (NRF- 2010-0022596) (S.K.). This work was supported by the Korea Research Foundation Grant funded by the Korean Government with grant numbers 2011-0009972 (O.K.), and by the World Class University grant no. R32-10130 (O.K.). This work was also supported by the National Research Foundation of Korea(NRF) grant funded by the Korea government(MEST) through the Center for Quantum Space- time(CQUeST) of Sogang University with grant number 2005-0049409 (P.O.) and by the BSRP through the National Research Foundation of Korea funded by the MEST (2010-21 0021996) (P.O.).

\section{References}

[1] A. H. Guth, "The Inflationary Universe: A Possible Solution to the Horizon and Flatness Problems," Phys. Rev. D 23, 347 (1981).

[2] D. H. Lyth, A. Riotto and, "Particle physics models of inflation and the cosmological density perturbation," Phys. Rept. 314, 1 (1999) hep-ph/9807278];

B. A. Bassett, S. Tsujikawa, D. Wands and , "Inflation dynamics and reheating," Rev. Mod. Phys. 78, 537 (2006) astro-ph/0507632;

D. Baumann, "TASI Lectures on Inflation," arXiv:0907.5424 [hep-th].

[3] P. A. R. Ade et al. [Planck Collaboration], "Planck 2013 results. XXII. Constraints on inflation," arXiv:1303.5082 [astro-ph.CO].

[4] G. Hinshaw, D. Larson, E. Komatsu, D. N. Spergel, C. L. Bennett, J. Dunkley, M. R. Nolta and M. Halpern et al., "Nine-Year Wilkinson Microwave Anisotropy Probe (WMAP) Observations: Cosmological Parameter Results," arXiv:1212.5226 [astro-ph.CO].

[5] J. M. Bardeen, "Gauge Invariant Cosmological Perturbations," Phys. Rev. D 22, 1882 (1980);

H. Kodama, M. Sasaki and, "Cosmological Perturbation Theory," Prog. Theor. Phys. Suppl. 78, 1 (1984);

V. F. Mukhanov, H. A. Feldman, R. H. Brandenberger and , "Theory of cosmological perturbations. Part 1. Classical perturbations. Part 2. Quantum theory of perturbations. Part 3. Extensions," Phys. Rept. 215, 203 (1992); 
A. R. Liddle, D. H. Lyth and, "The Cold dark matter density perturbation," Phys. Rept. 231, 1 (1993) astro-ph/9303019;

K. A. Malik, D. Wands and, Phys. Rept. 475, 1 (2009) [arXiv:0809.4944 [astro-ph]].

[6] J. M. Maldacena, "Non-Gaussian features of primordial fluctuations in single field inflationary models," JHEP 0305, 013 (2003) astro-ph/0210603;

N. Bartolo, E. Komatsu, S. Matarrese, A. Riotto and , "Non-Gaussianity from inflation: Theory and observations," Phys. Rept. 402, 103 (2004) astro-ph/0406398.

[7] D. Seery, J. E. Lidsey and , "Primordial non-Gaussianities from multiple-field inflation," JCAP 0509, 011 (2005) astro-ph/0506056;

G. I. Rigopoulos, E. P. S. Shellard, B. J. W. van Tent and , "Large non-Gaussianity in multiple-field inflation," Phys. Rev. D 73, 083522 (2006) astro-ph/0506704;

F. Vernizzi, D. Wands and , "Non-gaussianities in two-field inflation," JCAP 0605, 019 (2006) astro-ph/0603799;

D. Langlois, S. Renaux-Petel, D. A. Steer, T. Tanaka and, "Primordial perturbations and non-Gaussianities in DBI and general multi-field inflation," Phys. Rev. D 78, 063523 (2008) arXiv:0806.0336 [hep-th]];

X. Chen, Adv. Astron. 2010, 638979 (2010) [arXiv:1002.1416 [astro-ph.CO]].

[8] D. Polarski and A. A. Starobinsky, "Isocurvature perturbations in multiple inflationary models," Phys. Rev. D 50, 6123 (1994) astro-ph/9404061.

[9] M. Sasaki and E. D. Stewart, "A General analytic formula for the spectral index of the density perturbations produced during inflation," Prog. Theor. Phys. 95, 71 (1996) astro-ph/9507001.

[10] C. Gordon, D. Wands, B. A. Bassett and R. Maartens, "Adiabatic and entropy perturbations from inflation," Phys. Rev. D 63, 023506 (2001) astro-ph/0009131.

[11] C. Armendariz-Picon, "Creating Statistically Anisotropic and Inhomogeneous Perturbations," JCAP 0709, 014 (2007) [arXiv:0705.1167 [astro-ph]].

[12] S. Endlich, A. Nicolis and J. Wang, "Solid Inflation," arXiv:1210.0569 [hep-th].

[13] C. Omero and R. Percacci, "Generalized Nonlinear Sigma Models In Curved Space And Spontaneous Compactification," Nucl. Phys. B 165, 351 (1980). 
[14] M. Gell-Mann and B. Zwiebach, "Curling Up Two Spatial Dimensions With Su(1,1) / U(1)," Phys. Lett. B 147, 111 (1984); "Space-time Compactification Due To Scalars," Phys. Lett. B 141, 333 (1984).

[15] G. 't Hooft, "Unitarity in the Brout-Englert-Higgs Mechanism for Gravity," arXiv:0708.3184 [hep-th].

[16] K. Hinterbichler, "Theoretical Aspects of Massive Gravity," Rev. Mod. Phys. 84, 671 (2012) arXiv:1105.3735 [hep-th]].

[17] J. Lee, T. H. Lee, T. Y. Moon and P. Oh, "De-Sitter nonlinear sigma model and accelerating universe," Phys. Rev. D 80, 065016 (2009) [arXiv:0905.2653 [gr-qc]].

[18] C. M. Ho, T. W. Kephart and, "Inflatonless Inflation," Int. J. Mod. Phys. A 27, 1250151 (2012) arXiv:1002.4044 [hep-ph]].

[19] C. M. Ho, T. W. Kephart, D. Minic, Y. J. Ng and, "Spacetime Emergence and General Covariance Transmutation," Mod. Phys. Lett. A 28, 1350005 (2013) arXiv:1206.0085 [hepth]].

[20] M. Sasaki, "Large Scale Quantum Fluctuations in the Inflationary Universe," Prog. Theor. Phys. 76, 1036 (1986);

[21] J. Ohashi, J. Soda and S. Tsujikawa, "Anisotropic Non-Gaussianity from a Two-Form Field," Phys. Rev. D 87, 083520 (2013) [arXiv:1303.7340 [astro-ph.CO]].

[22] F. Lucchin and S. Matarrese, "Power Law Inflation," Phys. Rev. D 32, 1316 (1985);

J. D. Barrow, "Cosmic No Hair Theorems and Inflation," Phys. Lett. B 187, 12 (1987);

J. J. Halliwell, "Scalar Fields in Cosmology with an Exponential Potential," Phys. Lett. B 185, 341 (1987);

A. B. Burd and J. D. Barrow, "Inflationary Models with Exponential Potentials," Nucl. Phys. B 308, 929 (1988).

[23] A. A. Andrianov, F. Cannata and A. Y. .Kamenshchik, "General solution of scalar field cosmology with a (piecewise) exponential potential," JCAP 1110, 004 (2011) arXiv:1105.4515 $[\mathrm{gr}-\mathrm{qc}]]$.

[24] V. N. Lukash, "Production of phonons in an isotropic universe," Sov. Phys. JETP 52, 807 (1980) [Zh. Eksp. Teor. Fiz. 79]. 
[25] D. H. Lyth, "Large Scale Energy Density Perturbations and Inflation," Phys. Rev. D 31, $1792(1985)$;

V. F. Mukhanov, "Quantum Theory of Gauge Invariant Cosmological Perturbations," Sov. Phys. JETP 67 (1988) 1297 [Zh. Eksp. Teor. Fiz. 94N7 (1988) 1]. 